\title{
Downregulation of serum DKK-1 predicts poor prognosis in patients with papillary thyroid cancer
}

\author{
${ }^{1}$ Department of Nuclear Medicine, Affiliated Tumor Hospital, \\ Xinjiang Medical University, Ulumqi, China \\ 2Department of Thyroid Surgery, Affiliated Tumor Hospital, \\ Xinjiang Medical University, Ulumqi, China \\ ${ }^{3}$ Department of Clinical Laboratory, Affiliated Tumor Hospital, \\ Xinjiang Medical University, Ulumqi, China \\ ${ }^{4}$ Research Service Office, Affiliated Tumor Hospital, \\ Xinjiang Medical University, Ulumqi, China \\ Corresponding author: Y.P. Zhao \\ E-mail: yanping_zhaoxjmu@163.com
}

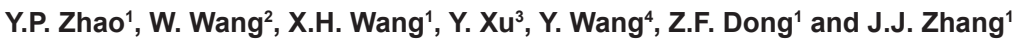

Genet. Mol. Res. 14 (4): 18886-18894 (2015)

Received August 15, 2015

Accepted October 14, 2015

Published December 28, 2015

DOI http://dx.doi.org/10.4238/2015.December.28.38

\begin{abstract}
The Wnt inhibitor dickkopf-1 (DKK-1) has been shown to be closely correlated with tumor initiation and progression in various types of cancers. However, the serum level of DKK-1 in patients with papillary thyroid cancer (PTC) and its potential clinical significance is poorly understood. Enzyme-linked immunosorbent assay (ELISA) was used to evaluate the level of serum DKK-1 in patients with PTC $(\mathrm{N}=132)$ and healthy controls $(\mathrm{N}$ $=40$ ). The association between serum DKK-1 level and clinicopathological parameters of PTC was examined and independent prognostic markers for PTC were identified. The mean serum DKK-1 level was significantly lower in patients with PTC than healthy controls $(44.64 \pm 15.13$ and $85.51 \pm 9.94$ $\mathrm{ng} / \mathrm{mL}$, respectively; $\mathrm{P}<0.01)$. Following treatment, the mean serum DKK1 level in PTC patients significantly increased $(67.03 \pm 17.09 \mathrm{ng} / \mathrm{mL} ; \mathrm{P}<$
\end{abstract}


0.01). Serum DKK-1 level was associated with various PTC clinical features including tumor size $(P=0.003)$, lymph node metastasis $(P=0.001)$, and tumor-node-metastasis stage $(P=0.004)$. Survival analysis revealed that PTC patients who had lower serum DKK-1 levels suffered both poorer overall survival $(P=0.036)$ and relapse-free survival $(P=0.015)$. Moreover, serum DKK-1 levels were an independent risk factor for predicting the prognosis of PTC ( $P=0.031)$. In conclusion, low DKK-1 serum levels are associated with poor prognosis in PTC patients and DKK-1 could potentially be used as a biomarker leading to earlier diagnosis of PTC.

Key words: DKK-1; Papillary thyroid cancer; Prognosis; Serum

\section{INTRODUCTION}

Thyroid cancer is the most common cancer of the endocrine system and its incidence has increased continuously in recent years. Papillary thyroid cancer (PTC) accounts for approximately $80 \%$ of all thyroid cancers and is the most common histological type (Lundgren et al., 2006). Patients with PTC generally have an excellent prognosis and are likely to be cured after treatment with surgery and/or radioiodinated therapy. However, recurrent disease develops in approximately 20 to $30 \%$ of PTC patients and roughly 7\% of PTC patients die within 10 years of diagnosis (Hundahl et al., 1998). Currently there is no specific and sensitive biomarker for monitoring therapy response or predicting the prognosis for PTC. Thus, exploring new biomarkers for PTC is of great significance to clinical practice.

The dickkopf (DKK) family encodes secreted proteins and is comprised of five members in vertebrates (DKK-1 to -4 , Soggy). The Wnt pathway plays an important role in regulation of the development of various diseases such as cancer, osteoporosis and cardiovascular diseases (Logan and Nusse, 2004; Pandey and Chandravati, 2013; Wang et al., 2014). DKK-1, a target of the $\beta$-catenin/TCF pathway, functions as a negative regulator of Wnt signaling. The dysregulation of DKK-1 has been shown to be associated with the initiation and progression of cancer. Chen et al. (2013) showed that DKK-1 was overexpressed in human hepatocellular carcinoma (HCC) where it acted as an oncogene in promoting the migration and invasion of HCC cancer cell lines, indicating that DKK-1 might be a target for therapy in HCC. Lee et al. (2012) reported that both tissue and serum DKK-1 levels in patients with gastric cancer were significantly higher in comparison with healthy controls. In addition, high serum DKK-1 was associated with poor survival. However, the role of DKK1 in carcinogenesis is complicated, as DKK-1 was also reported to be downregulated in various types of cancers. González-Sancho et al. (2005) reported that DKK-1 expression decreased in human colon cancer, suggesting that DKK-1 might play a role in tumor suppression in colon cancer.

DKK-1 levels were reported to be decreased in PTC tissues compared to that in normal tissues (Cho et al., 2014). However, the serum levels of DKK-1 in patients with PTC and its potential clinical significance are poorly understood. The aim of this study was to investigate the link between serum DKK-1 levels and PTC and determine if there is any potential prognostic value.

\section{MATERIAL AND METHODS}

\section{Study population}

The study was approved by the Research Ethics Committee of Affiliated Tumor Hospital, 
Xinjiang Medical University, and written consent was obtained from all PTC patients $(\mathrm{N}=132)$ and healthy volunteers $(N=40)$. Patients who received treatment from the Department of Thyroid Surgery were recruited in this study. The patients' diagnosis was confirmed by pathological evaluation after surgical removal of tumor tissues. The age of the patients ranged from 16 to 74 years and there were 47 males and 85 females. The clinical features of patients with PTC are summarized in Table 1. Healthy volunteers were individuals who generally had good health status and did not have thyroid-related diseases or other types of cancer.

\begin{tabular}{|c|c|c|c|c|}
\hline \multirow[t]{2}{*}{ Variable } & \multirow[t]{2}{*}{ No. of patients $(\mathrm{N})$} & \multicolumn{2}{|c|}{ Serum DKK-1 level (N) } & \multirow[t]{2}{*}{$P$ value } \\
\hline & & Low & High & \\
\hline \multicolumn{5}{|l|}{ Gender } \\
\hline Male & 47 & 19 & 28 & 0.239 \\
\hline Female & 85 & 51 & 34 & \\
\hline \multicolumn{5}{|l|}{ Age } \\
\hline$<45$ & 54 & 28 & 26 & 0.821 \\
\hline$\geq 45$ & 78 & 42 & 36 & \\
\hline \multicolumn{5}{|c|}{ Tumor size $(\mathrm{cm})$} \\
\hline$<1$ & 63 & 25 & 38 & 0.003 \\
\hline$\geq 1$ & 69 & 45 & 24 & \\
\hline \multicolumn{5}{|l|}{ Bilaterality } \\
\hline Unilateral & 82 & 42 & 40 & 0.594 \\
\hline Bilateral & 50 & 28 & 22 & \\
\hline \multicolumn{5}{|l|}{ Multifocality } \\
\hline Solitary & 67 & 37 & 30 & 0.608 \\
\hline Multiple & 65 & 33 & 32 & \\
\hline \multicolumn{5}{|c|}{ Lymph node metastasis } \\
\hline No & 71 & 28 & 43 & 0.001 \\
\hline Yes & 61 & 42 & 19 & \\
\hline \multicolumn{5}{|c|}{ Extrathyroidal extension } \\
\hline No & 76 & 41 & 35 & 0.806 \\
\hline Yes & 56 & 29 & 27 & \\
\hline \multicolumn{5}{|l|}{ TNM stage } \\
\hline $\mathrm{I}-\mathrm{II}$ & 93 & 42 & 51 & 0.004 \\
\hline III-IV & 39 & 28 & 11 & \\
\hline
\end{tabular}

\section{Enzyme-linked immunosorbent assay (ELISA)}

Freshly isolated whole venous blood was collected (up to $6 \mathrm{~mL}$ ) from each participant, and the serum was separated from the blood by centrifugation at $1000 \mathrm{~g}$ for $5 \mathrm{~min}$ at room temperature followed by centrifugation at $12,000 \mathrm{~g}$ at $4^{\circ} \mathrm{C}$ for $5 \mathrm{~min}$. The serum samples were stored at $-80^{\circ} \mathrm{C}$ until use. Serum DKK-1 levels were detected by ELISA with immunoassay kit (Biomedica, Vienna, Austria) according to the manufacturer instructions. Serum $(25 \mu \mathrm{L})$ was incubated with $50 \mu \mathrm{L}$ DKK1 antibody for $2 \mathrm{~h}$ at room temperature. After the plates were washed with PBS, the conjugate was then added, and the plates were incubated for another $2 \mathrm{~h}$. Finally, the substrate was added and incubated for 30 minutes, and the optical density (OD) at $450 \mathrm{~nm}$ was determined.

\section{Statistical analysis}

The levels of serum DKK-1 in patients with PTC and healthy controls were compared using the Mann-Whitney U-test. Chi-square tests were then employed to evaluate the correlation 
between serum DKK-1 level and various important clinicopathological parameters of PTC. Kaplan-Meier analysis was performed to assess both overall survival and relapse free survival, and multivariate analysis was conducted using the Cox proportional hazard model to identify the independent prognostic factors for PTC. All statistical analyses were performed using SPSS 21 software package (SPSS Inc., Chicago, IL, USA). A P value of less than 0.05 was considered statistically significant.

\section{RESULTS}

\section{Level of DKK-1 in serum from patients with PTC and healthy controls}

Our ELISA results showed that the mean concentration of serum DKK-1 in patients with PTC and healthy volunteers was $44.64 \pm 15.13 \mathrm{ng} / \mathrm{mL}$ and $85.51 \pm 9.94 \mathrm{ng} / \mathrm{mL}$, respectively. The expression level of serum DKK-1 in patients with PTC was significantly lower than in healthy controls $(P<0.01)$ (Figure 1).

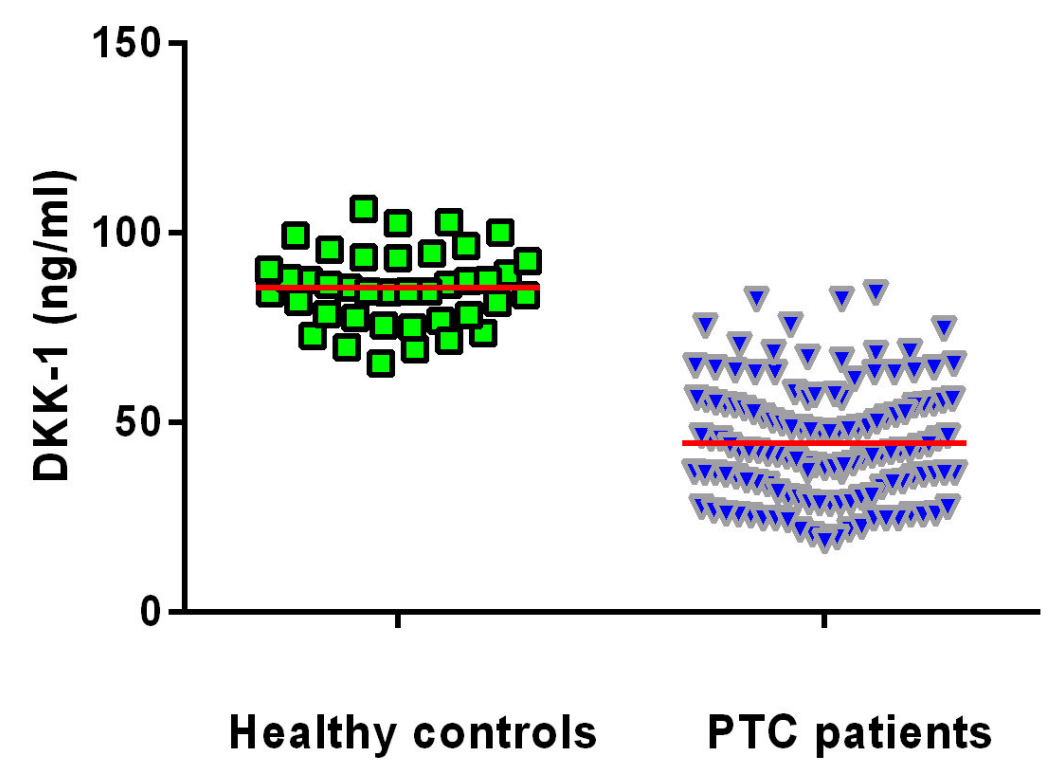

Figure 1. Expression level of serum DKK-1 in patients with PTC and healthy controls.

\section{Level of DKK-1 in serum from patients with PTC after receiving therapy}

Our results showed that the mean concentration of serum DKK-1 was significantly increased in PTC patients who received treatment, including surgery and radioiodinated therapy, compared to PTC patients who didn't receive treatment $(67.03 \pm 17.09$ and $44.64 \pm 15.13 \mathrm{ng} / \mathrm{mL}$, respectively; $P<0.01$ ) (Figure 2). This indicates that serum DKK-1 level may be correlated with treatment response. 


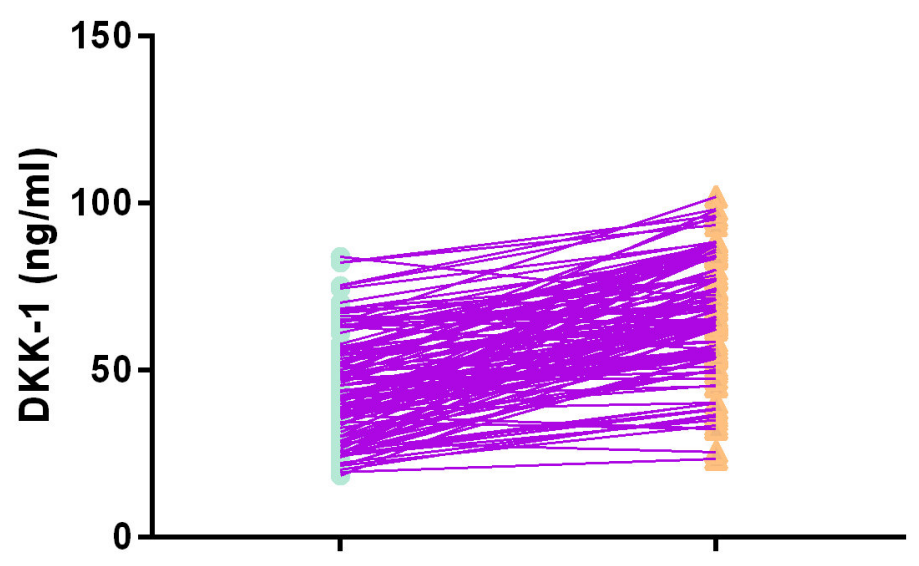

\section{Before therapy After therapy}

Figure 2. Expression level of DKK-1 in serum samples from PTC patients was significantly increased after receiving therapy.

\section{Association between serum DKK-1 level and clinicopathological parameters of PTC}

The median level of serum DKK-1 in PTC patients was $42.6 \mathrm{ng} / \mathrm{mL}$, which was used to divide the patients into two groups (high and low serum DKK-1 group). Our results revealed that serum DKK-1 level was closely correlated with various important clinical features including tumor size $(P=0.003)$, lymph node metastasis $(P=0.001)$, and tumor-node-metastasis $(T N M)$ stage $(P$ $=0.004)$. However, it was not associated with sex $(P=0.239)$, age $(P=0.821)$, bilaterality $(P=$ $0.594)$, multifocality $(P=0.608)$ and extrathyroidal extension $(P=0.806)($ Table 1$)$.

\section{Low serum DKK-1 level is associated with poor prognosis in PTC}

The survival analysis showed that the PTC patients in the low serum DKK-1 group had both poorer overall survival $(P=0.036)$ and relapse-free survival $(P=0.015)$ in comparison with the patients in the high serum DKK-1 group (Figures 3 and 4). Our multivariate analysis showed that tumor size $(P=0.024)$, lymph node metastasis $(P=0.008)$, TNM stage $(P=0.012)$ and serum DKK-1 level $(P=0.031)$ were independent risk factors for PTC (Table 2).

\section{DISCUSSION}

There are four main types of thyroid cancer: PTC, follicular thyroid cancer, medullary thyroid cancer, and anaplastic thyroid cancer. The prognosis and treatment effects are closely correlated with the histological type of cancer and tumor stage (Nguyen et al., 2015). With the development of advanced screening methods, the incidence of PTC has increased dramatically in recent years. PTC has a generally indolent character and the prognosis is favorable. However, radioiodine refractory metastatic/recurrent PTC remains a clinical challenge (Kim, 2015). Thus, 


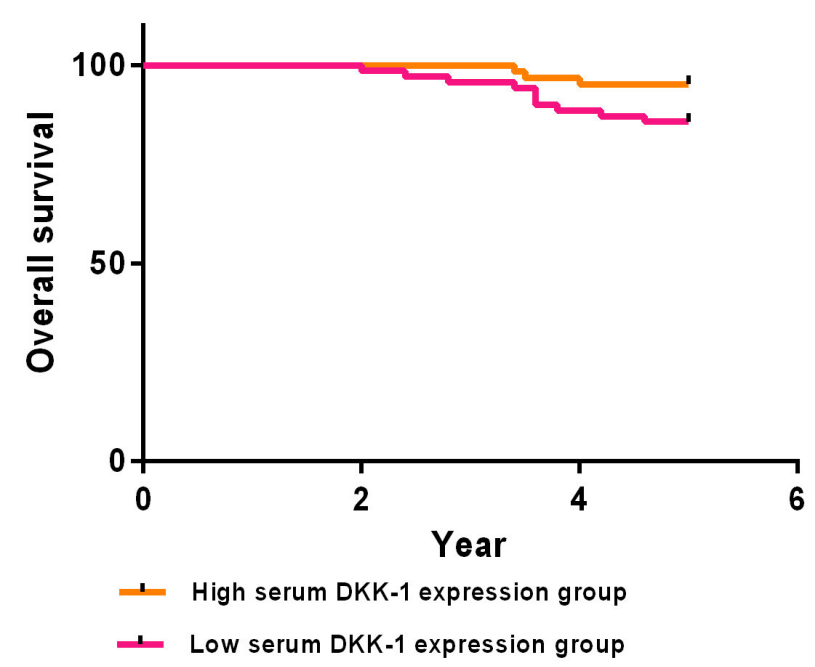

Figure 3. The PTC patients in the low serum DKK-1 group had poorer overall survival rate.

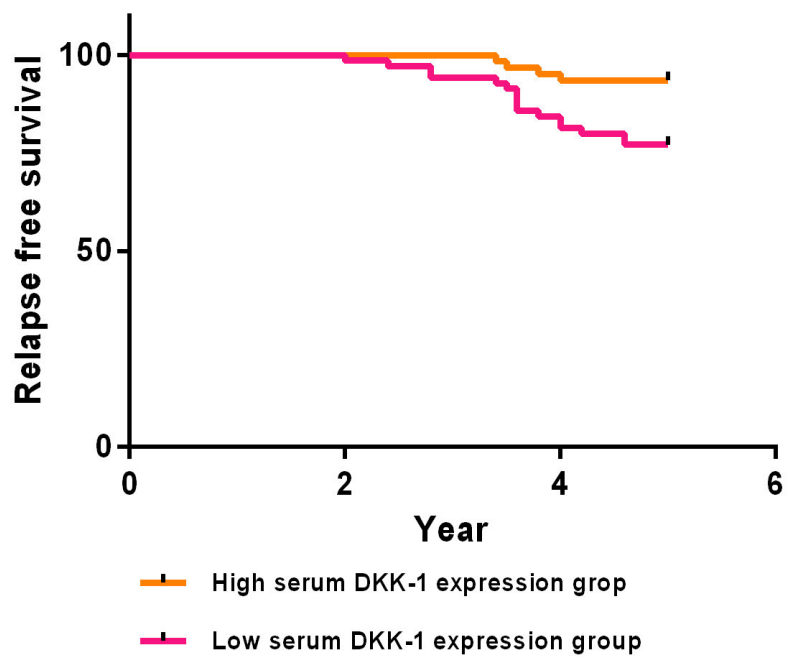

Figure 4. PTC patients in the low serum DKK-1 group suffered poorer relapse free survival rate.

\begin{tabular}{lcc}
\multicolumn{2}{l}{ Table 2. The prognostic factors of PTC. } & \\
\hline Parameter & Hazard ratio & P value \\
\hline Gender (Female vs Male) & 1.19 & 0.712 \\
Age $(\geq 45$ vs $<45$ ) & 1.41 & 0.247 \\
Tumor size $(\geq 1$ cm vs $<1$ cm) & 2.64 & 0.024 \\
Bilaterality (Bilateral vs Unilateral) & 1.32 & 0.465 \\
Multifocality (Multiple vs Solitary) & 1.22 & 0.615 \\
Lymph node metastasis (Yes vs No) & 3.25 & 0.008 \\
Extrathyroidal extension (Yes vs No) & 1.05 & 0.889 \\
TNM stage (III-IV vs I-II) & 2.93 & 0.012 \\
Serum DKK-1 level (Low vs High) & 2.48 & 0.031 \\
\hline
\end{tabular}


diagnosis of PTC at an earlier stage is extremely important.

As a negative regulator of the Wnt signaling pathway, DKK-1 binds to low-density lipoprotein receptor-related protein 5/6 (LRP5/6) and blocks its interaction with Wnt-1, resulting in $\beta$-catenin degradation and effects on proliferation (Semënov et al., 2001). Qiao et al. (2008) showed that DKK-1 is secreted by mesenchymal stem cells, inhibiting growth of breast cancer cells through inhibition of Wnt signaling. In addition to the canonical Wnt signaling pathway, DKK-1 might be also involved in regulation of the non-canonical Wnt pathway and other signaling pathways such as the c-Jun N-terminal kinase pathway (Peng et al., 2006; Koppen et al., 2007). As DKK-1 plays important roles in many essential pathways that are related to cell proliferation, migration, and survival, it is not surprising that its dysregulation was detected in many cancers. One of the advantages of diagnosis from body fluids rather than with biopsy tissues is that therapy response can be monitored and prognosis can be predicted in real time. In addition, collection of body fluids is significantly less invasive than obtaining a biopsy.

In the present study, our results showed that patients with PTC had lower levels of serum DKK-1 compared with healthy volunteers. In addition, the PTC patients' serum DKK-1 levels were significantly higher after they received therapy. Serum DKK-1 level was associated with a variety of clinical parameters including tumor size, lymph node metastasis, and TNM stage. Low serum DKK-1 level indicated both poorer overall survival and relapse-free survival. Additionally, serum DKK-1 was an independent risk factor for predicting the prognosis of PTC. Consistent with our findings, Cho et al. (2014) reported that DKK-1 was downregulated in PTC tissues in comparison with normal tissues. Ectopic overexpression of DKK-1 in PTC cell lines could inhibit tumor growth significantly, indicating that DKK-1 might be a therapeutic target for PTC. The same group also showed that DKK-1 suppressed the survival and migration of human PTC cells by regulating Wnt/ $\beta$-catenin signaling and E-cadherin expression (Cho et al., 2013). In regards to PTC, we hypothesized that DKK-1 might play a tumor suppressive role. Zhou et al. (2010) revealed that DKK-1 was downregulated in LM-MCF-7 (a cell line with a higher proliferative and metastatic capacity) relative to MCF-7 cells. In addition, upregulation of DKK-1 could inhibit the proliferation of breast cancer cell lines by downregulating the expression of $\beta$-catenin, c-Myc, cyclin D1, and survivin (Zhou et al., 2010). The level of DKK-1 in tobacco smoke condensate (TSC)-induced lung cancer cell lines was decreased compared to controls, and inhibition of DKK-1 in vitro could mimic the pro-tumorigenic effects of TSC exposure (Hussain et al., 2009). Kuphal et al. (2006) revealed that the level of DKK-1 was downregulated in melanoma tissues and cancer cells lines. Moreover, the level of serum DKK-1 was deceased in advanced stage melanoma, suggesting that decreases in serum DKK-I could be a sign that control of tumor growth has been lost (Feldmann et al., 2011).

However, the role of DKK-1 varies in different types of cancers. In some cases, the expression of DKK-1 has been shown to be upregulated in various kinds of cancers. Rachner et al. (2014) showed that both tissue and serum DKK-1 levels were significantly higher in prostate cancer patients compared with healthy controls. In addition, high serum DKK-1 levels were shown to be an independent prognostic factor for prostate cancer, suggesting that DKK-1 might act as an oncogene in prostate cancer. Takahashi et al. (2010) showed that DKK-1 was overexpressed in both human pancreatic ductal adenocarcinoma tissues and cell lines, and knockdown of DKK-1 could inhibit the proliferative and invasive capability of human pancreatic ductal adenocarcinoma cell lines in vitro. Similarly, the serum level of DKK-1 was elevated in patients with osteosarcoma, and the expression of DKK-1 by the osteosarcoma cells at the periphery of the tumor might be responsible for tumor growth by inhibiting repair of the surrounding bone (Lee et al., 2007). 
Since DKK-1 plays opposing roles in different types of cancers, it is possible that its function might be tissue-dependent. However, contradictory findings on the role of DKK-1 in the same type of cancer have also been reported. Mikheev et al. (2008) showed that DKK-1 was a tumor suppressor in breast cancer both in vitro and in vivo. However, high serum DKK-1 levels were detected in patients with breast cancer and it was associated with poor prognosis, indicating that DKK-1 could promote tumorigenesis in breast cancer (Zhou et al., 2014). One possible explanation for these results is that the precise role of DKK-1 might be influenced by the tumor microenvironment. Further studies are needed to address the complex role of DKK-1 in regulation of tumor initiation and progression. The serum level of DKK-1 was decreased in patients with PTC and its downregulation was associated with poor prognosis, indicating that serum DKK-1 levels could be used as a noninvasive biomarker for the prognosis of PTC.

\section{Conflicts of interest}

The authors declare no conflict of interest.

\section{ACKNOWLEDGMENTS}

We would like to thank doctors in the Department of Thyroid Surgery, the Staff at the Department of Clinical Laboratory, Affiliated Tumor Hospital of Xinjiang Medical University, for technical and clinical support.

\section{REFERENCES}

Chen L, Li M, Li Q, Wang CJ, et al. (2013). DKK1 promotes hepatocellular carcinoma cell migration and invasion through ß-catenin/MMP7 signaling pathway. Mol. Cancer 12: 157.

Cho SW, Lee EJ, Kim H, Kim SH, et al. (2013). Dickkopf-1 inhibits thyroid cancer cell survival and migration through regulation of $\beta$-catenin/E-cadherin signaling. Mol. Cell. Endocrinol. 366: 90-98.

Cho SW, Kim YA, Sun HJ, Ahn HY, et al. (2014). Therapeutic potential of Dickkopf-1 in wild-type BRAF papillary thyroid cancer via regulation of $\beta$-catenin/E-cadherin signaling. J. Clin. Endocrinol. Metab. 99: e1641-e1649.

Feldmann R, Schierl M, Fink AM, Sator PG, et al. (2011). Serum levels of glycoprotein Dickkopf-1 in patients with cutaneous malignant melanoma: a prospective pilot study. Dermatology 222: 171-175.

González-Sancho JM, Aguilera O, García JM, Pendás-Franco N, et al. (2005). The Wnt antagonist DICKKOPF-1 gene is a downstream target of beta-catenin/TCF and is downregulated in human colon cancer. Oncogene 24: 1098-1103.

Hundahl SA, Fleming ID, Fremgen AM and Menck HR (1998). A National Cancer Data Base report on 53,856 thyroid carcinoma treated in the U.S., 1985-1995 [see comments]. Cancer 83: 2638-2648.

Hussain M, Rao M, Humphries AE, Hong JA, et al. (2009). Tobacco smoke induces polycomb-mediated repression of Dickkopf-1 in lung cancer cells. Cancer Res. 69: 3570-3578.

Kim WB (2015). A closer look at papillary thyroid carcinoma. Endocrinol. Metab. 30: 1-6.

Koppen A, Ait-Aissa R, Hopman S, Koster J, et al. (2007). Dickkopf-1 is down-regulated by MYCN and inhibits neuroblastoma cell proliferation. Cancer Lett. 256: 218-228.

Kuphal S, Lodermeyer S, Bataille F, Schuierer M, et al. (2006). Expression of Dickkopf genes is strongly reduced in malignant melanoma. Oncogene 25: 5027-5036.

Lee HS, Lee HE, Park do J, Kim HH, et al. (2012). Clinical significance of serum and tissue Dickkopf-1 levels in patients with gastric cancer. Clin. Chim. Acta 413: 1753-1760.

Lee N, Smolarz AJ, Olson S, David O, et al. (2007). A potential role for Dkk-1 in the pathogenesis of osteosarcoma predicts novel diagnostic and treatment strategies. Br. J. Cancer 97: 1552-1559.

Logan CY and Nusse R (2004). The Wnt signaling pathway in development and disease. Annu. Rev. Cell Dev. Biol. 20: 781-810.

Lundgren Cl, Hall P, Dickman PW and Zedenius J (2006). Clinically significant prognostic factors for differentiated thyroid carcinoma: a population-based, nested case control study. Cancer 106: 524-531. 
Mikheev AM, Mikheeva SA, Maxwell JP, Rivo JV, et al. (2008). Dickkopf-1 mediated tumor suppression in human breast carcinoma cells. Breast Cancer Res. Treat. 112: 263-273.

Nguyen QT, Lee EJ, Huang MG, Park YI, et al. (2015). Diagnosis and treatment of patients with thyroid cancer. Am. Health Drug Benefits 8: 30-40.

Pandey S and Chandravati (2013). Targeting Wnt-Frizzled signaling in cardiovascular diseases. Mol. Biol. Rep. 40: $6011-6018$.

Peng S, Miao C, Li J, Fan X, et al. (2006). Dickkopf-1 induced apoptosis in human placental choriocarcinoma is independent of canonical Wnt signaling. Biochem. Biophys. Res. Commun. 350: 641-647.

Qiao L, Xu ZL, Zhao TJ, Ye LH, et al. (2008). Dkk-1 secreted by mesenchymal stem cells inhibits growth of breast cancer cells via depression of Wnt signalling. Cancer Lett. 269: 67-77.

Rachner TD, Thiele S, Göbel A, Browne A, et al. (2014). High serum levels of Dickkopf-1 are associated with a poor prognosis in prostate cancer patients. BMC Cancer 14: 649.

Semënov MV, Tamai K, Brott BK, Kühl M, et al. (2001). Head inducer Dickkopf-1 is a ligand for Wnt coreceptor LRP6. Curr. Biol. 11: 951-961.

Takahashi N, Fukushima T, Yorita K, Tanaka H, et al. (2010). Dickkopf-1 is overexpressed in human pancreatic ductal adenocarcinoma cells and is involved in invasive growth. Int. J. Cancer 126: 1611-1620.

Wang Y, Li YP, Paulson C, Shao JZ, et al. (2014). Wnt and the Wnt signaling pathway in bone development and disease. Front. Biosci. Landmark Ed. 19: 379-407.

Zhou SJ, Zhuo SR, Yang XQ, Qin CX, et al. (2014). Serum Dickkopf-1 expression level positively correlates with a poor prognosis in breast cancer. Diagn. Pathol. 9: 16.

Zhou XL, Qin XR, Zhang XD and Ye LH (2010). Downregulation of Dickkopf-1 is responsible for high proliferation of breast cancer cells via losing control of Wnt/beta-catenin signaling. Acta Pharmacol. Sin. 31: 202-210. 